\title{
Effectiveness of Educational Guidelines Adherence on Health Complaints and Anxiety Level among Patients with Coronary Artery Disease
}

\author{
Ola Abdelwahab Abdallah Srour ${ }^{1}$, Shimaa Attia Ali ${ }^{2}$ \\ ${ }^{1,2}$ Lecturer of Adult Nursing, Faculty of Nursing, Helwan University, Egypt
}

\begin{abstract}
Coronary artery disease is the leading cause of death globally and the most common type of heart disease. Health complaints are subjective in nature; it may afford information on the degree of recovery from coronary artery disease. Educational guidelines play a vital role in increasing knowledge, practice, and modifying health behaviors. This study aimed to evaluate the effect of educational guideline adherence on health complaints and anxiety level among patients with coronary artery disease. Subjects and Method: Design: Quasi-experimental research design. Setting: The study was conducted at cardiology and coronary care unit affiliated with El Hussein University Hospitals. Subjects: A Purposive sample of 104 adult patients was selected and equally divided into (52) study and (52) control group. Three Tools include Patients' Structured Interview Questionnaire, The Somatic Health Complaints Questionnaire and Beck Anxiety Inventory Questionnaire was used in this study. Results: The mean age of the study and control group were $(54.21 \pm 8.01 \& 54.71$ \pm 10.42 , respectively), $59.6 \%$ of both groups were males. There was a highly statistically significant difference between study and control group regarding total mean scores of knowledge, all dimensions of health complaints and anxiety level scores $(\mathrm{p}<0.001)$ at post and follows up phases throughout implementation. Conclusion: There was a highly statistically significant improvement regarding the mean scores of total knowledge, somatic health complaints and anxiety level for the study group at the post and follow up phases of the educational guidelines Adherence implementation. Recommendation: Develop Telecommunication interventions in secondary prevention for patients with CAD to promote effective reduction of risk factors.
\end{abstract}

Key words: Coronary artery disease, Educational guidelines, Health complaints, Anxiety Level, Adherence. 


\section{Introduction}

Coronary Artery Disease (CAD) has become the largest single cause of death globally. It is responsible for a huge global economic burden and a large percentage of this burden is found in low and middle income countries. Coronary artery disease is the prominent reason for mortality within the United States. It is the third leading cause of mortality worldwide and is attendant with 17.8 million deaths annually. While, CAD is a significant cause of death and disability, it is preventable $^{(1,2)}$.

Most studies have categorized the risk factors for $\mathrm{CAD}$ into non-modifiable (Age, gender, ethnicity, genetic factors and family history) and modifiable risk factors either behavioral (Unhealthy food, physical inactivity, smoking and alcohol intake) or physical factors (Diabetes mellitus, hypertension, dyslipidemia, increased waist circumference, and improper body mass index, with the contribution of psychological /psychosocial factors (Anxiety and stress) ${ }^{(2,3)}$.

Coronary artery disease could be marked stable ischemic heart disease (SIHD) or acute coronary syndrome (ACS). It can be more advanced into congestive heart failure (CHF) if not controlled. Physical symptoms include chest pain, radiation of the pain in the jaw, neck, left arm, or into the back. Dyspnea should be evaluated at rest and also on the activity, jugular venous distention, palpitations, tachypnea, lower extremity edema and orthopnea. Patient inspected for any acute distress, and peripheral edema, this has a significant influences on health care and nursing practice $^{(4,5,6)}$.

Although, health complaints are subjective in nature, it may afford statistics on the degree of rescue from coronary heart disease (CHD). Obviously, within the weeks from a coronary incident, patients repeatedly complained from somatic health complaints focused on (Chest pain, dyspnea, sleep problems, fatigue). And also, cognitive health complaints concern about (cognitive functional status and anxiety level) ${ }^{(5,7-9)}$.

On the top, patients with CAD are more prospective to suffer from psychological problems like anxiety or depression because they usually tolerate unpleasant symptoms without warning and are required to take numerous categories of medications throughout their whole lifespan, resulting in negative emotions. The percentage of patients concerned by anxiety and depression was recounted to be raised to $15-43 \%$ during the first 12 months after an acute cardiac incident ${ }^{(10,11)}$.

Anxiety is accompanying with minor diagnosis in patients with coronary artery 
disease (CAD). Moreover, patients with high anxiety level had non-adherence to cardiac medication with non-attendance and non-completion of cardiac rehabilitation programs. Consequently, it has a negative influence of on health performances and adherence to evidence based hazard decreasing guidelines and managements for $\mathrm{CAD}^{(12)}$.

European Society of Cardiology (ESC) guidelines for the management of coronary syndromes were designed to achieve optimal disease stabilization or regression and included pharmacological management, developing healthy lifestyle behaviors, and percutaneous coronary interventions. A multidisciplinary team an approach that provides personalized and flexible support to patients can lead to the achievement of optimum management outcomes ${ }^{(13)}$.

Educational guidelines adherence are relevant to the patient's level of understanding and comprehension, delivered at the appropriate time, as well as it can be one of the fundamentals for the patient to raise their knowledge, practice and awareness of the significance of making determinations about medical management and improve the patient's outcomes. Extensive education has the main role in lowering the risk of coronary heart disease. In combination with the outcomes from other studies, rising education is expected to improve health benefits $(14,15,16)$.

\section{Significance of the study}

Globally, CAD pretends to have about 126 million individuals and the recent prevalence rate of 1,655 per 100,000 populations is predictable to outstrip 1,845 by the year 2030. Which is approximately $1.72 \%$ of the world's population and about 9 million deaths were affected by CAD. Commonly, women were lesser affected than men, and occurrence typically ongoing in the fourth decade and prognosis with age ${ }^{(17)}$. Concerning to the most recent World Health Organization Rankings data published the coronary heart disease deaths in Egypt reached 271.69 per 100,000 of population ranks which about $29.38 \%$ of total deaths rate as well as Egypt is considered number 15 in the world rank ${ }^{(18)}$.

The World Health Organization shared a comprehensive plan of action for the prevention and control of noncommunicable diseases and cardiovascular disorders to take actions to empower patients in managing their health conditions, promote education, encouragements and tools for self-care and self-management that are based on 
guidelines and patient records, and also a team based on patient management ${ }^{(19,20,21)}$. Thus, health education for patients with $\mathrm{CAD}$ is vital elements that make contributions in the controlling of patients' health condition and secondary prevention of disease. So the aim of this study was to evaluate effect of educational guidelines adherence on health complaints and anxiety level among patients with coronary artery disease

\section{Aim of the Study}

The aim of this study was to evaluate the effect of educational guideline adherence on health complaints and anxiety level among patients with coronary artery disease through:

1. Assessing patients' level of knowledge, patients' health complaint and anxiety level.

2. Developing educational guideline according to patients' health complaints and anxiety level.

3. Implementing educational guideline according to patients' health complaints and anxiety level.

4. Evaluating the effect of an educational guideline adherence on patient's knowledge, patient's health complaints and anxiety level.

\section{Hypotheses:}

The current study hypothesized that:
-Study group will have a statistically significant improvement in knowledge level post educational guideline adherence as compared to control group.

-Study group will have a statistically significant fewer health complaints and anxiety level post educational guideline adherence as compared to control group.

\section{Subjects and methods}

Research design: Quasi-experimental research design was utilized to achieve the aim of this study.

\section{Technical Design:}

The technical design includes research setting, subjects and tools for data collection.

Setting: The study was conducted in Cardiology Unit and Cardiac Care Unit at El Hussein University Hospital.

Subjects: A Purposive sample of (104) adult patients diagnosed with CAD, was selected and equally divided into the study and control group, (52) patients for each one. The sample size is determined by statistical analysis (power analysis) where it represents the total number of patients who are diagnosed with $\mathrm{CAD}$ in the cardiology unit at El Hussein University Hospital. At year 2018 which were (1044) patients. 


\section{Inclusion Criteria:}

Adult patients above 18 years, from both sexes who agree to participate in the study, not exposed before to any teaching or learning experiences regarding coronary artery diseases.

\section{Exclusion Criteria:}

Patients with mental problems were excluded.

\section{Tools for data Collection:}

Data for this study were collected using the following tools:

\section{A-Patient Structured Interview}

Questionnaire: An Arabic questionnaire was developed by the researchers, after reviewing the related literature and research studies $(3,22,23)$. It included the following parts:

Part 1: Demographic characteristics: it included age, sex, residence, level of education and occupation.

Part 2: Past Medical History/life style: It included series of questions to elicit patient's past medical history, compliance to medications, and smoking.

\section{Part 3: Patient learning needs} assessment sheet: It was developed by the researchers based on relevant and recent literatures ${ }^{(24)}$. It was used to assess the knowledge level of patients with CAD. It contains true \& false and multiple choice questions (23) items including; definition of disease, causes, risk factors, signs and symptoms, diagnostic tests, management and disease related instructions.

\section{Scoring system:}

The answer was evaluated using model key answer prepared by the researcher. The score 2 gave for correct answers, and 1 for an incorrect answer. The total knowledge score was (46).

The level of the patient's knowledge was considered unsatisfactory when less than $60 \%$, while $\geq 60 \%$, the patient level of knowledge was considered as satisfactory level.

\section{Tool II: The Somatic Health Complaints}

\section{Questionnaire (SHCQ):}

- This questionnaire was adapted from Brink et al. (2007) ${ }^{(25)}$. The questionnaire addresses 13 health problems common in cardiac patients: chest pain, chest pain that limits daily activity, shortness of breath, shortness of breath during exertion, sweating, dizziness, headache, and stomach trouble, lack of energy, heart palpitations, tiredness, weakness, and sleep disturbance. The patients were asked to report how often these symptoms had occurred during the past week. Each question was answered using a 6-point Likert scale, Translation and back translation from English to Arabic was done for this tool to assure accuracy for content validity. 


\section{- Scoring system:}

The patient response for each statement was made on a 6-point Likert scale, ranging from ( 1 to 6 ) as follows: $1=$ never, 2=once, 3=sometimes, 4=several times, $5=$ mostly, $6=$ always.

\section{Tool III: Beck Anxiety Inventory:}

This tool adopted from Beck and Steer $(\mathbf{1 9 9 0})^{(26)}$, it is a 21 -items multiple-choice self-report inventory that measures the severity of an anxiety in cardiac patients. Translation and back translation from English to Arabic was done for this tool to assure accuracy for content validity.

\section{Scoring system:}

Each symptom item has four possible answer choices: Not at All; Mildly (It did not bother me much); moderately (It was very unpleasant, but I could stand it), and; severely (I could barely stand it). The clinician assigns the following values to each response: Not at All $=0$; mildly $=1$; moderately $=2$, and; severely $=3$. The values for each item are summed yielding an overall or total score for all 21 symptoms that can range between 0 and 63 points. A total score of $0-7$ is interpreted as a "Minimal" level of anxiety; 8 - 25 as "Moderate" and 26 - 63 as "Severe".

\section{Proposed educational guidelines:}

These educational guidelines were developed for patients with CAD to enrich them with information related to CAD, and measures to overcome complications, nutritional management and importance of a healthy diet, and measures to improve patient's level of knowledge, health complaints, and anxiety level.

\section{Operational Design:}

The operational design includes preparatory phase, pilot study and field work.

\section{Preparatory Phase:}

It included appraising of current and past available theoretical knowledge of different features of the study using booklet, articles, internet, periodicals and magazines in order to develop the data collection tools.

\section{Content validity:}

Content validity was conducted to test the tool for appropriateness, relevance, correction and clearance through a jury of seven experts, from the medical-surgical nursing staff at the faculty of nursing, Helwan University. Juries were from different academic categories (professors and assistant professors). Their opinions were elicited regarding the tool format, layout and consistency and scoring system.

\section{Pilot Study:}

A pilot study was applied to a group of 10 patients (10\% of the sample) to test the applicability of the tools and clarity of the designed questionnaire, as well as to estimate the time desirable to answer them. Patients included in the pilot study were 
also included in the main study subject, because there were no modifications in the tools.

\section{Reliability:}

- It is tested by using Cronbach alpha test the reliability scores of study tools including Arabic version, for tool II, III were $(0.82$ and 0.81$)$ for the Somatic Health Complaints Questionnaire (SHCQ) and Beck Anxiety Inventory respectively.

\section{Field Work:}

- Data collection was started and completed within 6 months from beginning of July 2019 until end of December 2019.

- Purpose of the study was explained by the researchers to patients who agreed to participate in the study prior to any data collection; the study sample divided into the study and control group.

- The study tools were filled in and completed by the researchers three times on 3 stages (pre \& post and follow up after three months from guidelines implementation phases).

- The researchers were available at cardiology unit \& the cardiac care unit at El Hussein university hospital 3 days/week at morning and afternoon shifts to collect data from the studied patients. Filling in the tools was done according to the patients` understanding and health condition.

The collection of data was done through three phrases:
Assessment phase: In this phase the researchers collected data from both groups (study and control) starting with control group to prevent contamination of the sample. The time needed for completing study tools was about (20-35 minutes) for each patient.

\section{Implementation phase:}

In this phase the developed educational guidelines were implemented by the researchers in the study group only. The researchers started to discuss educational guidelines with the patient explaining the aim and objectives of the program as well as distributing booklet and explaining its content .The educational guidelines were delivered in Cardiology Unit for every 3 to 4 patients together according to their education level and understanding. The educational guidelines were supported by using posters, power points, colored booklet and lectures as different strategies for educating patients. Patients were allowed to ask questions in case of misunderstanding, while listening and expressing interest for them. At the end of the program sessions the researchers emphasized the importance of the follow up visits and informed the patients to be in contact with them by telephone in case of questions related to their condition and informed them that they will be followed up by the researchers after three months. 
Evaluation phase: This phase was conducted through interviewing patients at the outpatients' clinic post immediately \& after three months (follow up) by using the same tools to evaluate the effect of the implemented educational guideline on patient's knowledge, health complaints and anxiety level. It was tested by comparing the results of the data collected post immediately \& after three months from the study and control groups.

\section{Administrative Design:}

To carry out the study, the necessary approvals were obtained from the hospital director and nursing director of El Hussein University Hospital. Official letters were issued to them from the Faculty of Nursing explaining the aim of the study to obtain permission for the collection of data.

\section{Ethical Considerations:}

A formal consent was taken from patients who agree to participate in the research process after the aim of the study has been simply explained to them prior to data collection. They were assured that anonymity and confidentiality would be guaranteed and the right to withdraw from the study at any time without giving any reason. Values, culture and beliefs would be respected.

Statistical Design: Data collection obtained, they were organized, categorized, tabulated and analyzed. Data were presented in tables, figures and diagram using the Statistical Package for Social Science (SPSS). Statistical significant associations were assessed using percentage $(\%)$, mean, standard deviation, t-test, chi square and p-value.

\section{Results}

Table (I) Demographic characteristics among the study and control group subjects illustrated that, $53.8 \%$ of the study group and $36.5 \%$ of the control group had the same age from 50 years to less than 60 years old with mean age $(54.21 \pm 8.01$ and $54.71 \pm 10.42$, respectively). Regarding sex, $59.6 \%$ of study and control groups were males. Concerning the residence, $84.6 \%$ of the study group and $67.3 \%$ of the control group were living in urban areas. As regards to level of education, $44.2 \%$ of the study group and $51.9 \%$ of control group can read and write. About patient's occupation, $26.9 \%$ of the study group and $34.6 \%$ of the control group had muscular work. While, $44.2 \%$ and $46.2 \%$ of study and control groups, respectively, were not working. The results showed also, that there were no statistically significant differences between study and control group subjects regarding all aspects of demographic characteristics at $\mathrm{p}<0.05$.

Figure (1) Percentage distribution of the studied group by marital status showed that, marital status, $71.2 \%$ and 76.9 of 
study and control groups respectively were married.

Table (2) Percentage distribution among the study and control group subjects as regards health history indicated that $42.3 \%$ and $48.1 \%$ of study and control groups, respectively had diabetes while, $73.1 \%$ and $76.9 \%$ of study and control groups, respectively had hypertension. Moreover, $59.6 \%$ and $63.5 \%$ of the study and control groups, respectively had angina. $26.9 \%$ of study group and $51.9 \%$ of the control group had IHD. It is obvious from the above table that $25.0 \%$ of the study group and $36.5 \%$ of the control group had previous history of cardiac catheterization. However, $80.8 \%$ and $76.9 \%$ of the study and control groups respectively had incomplete compliance with medication. Concerning smoking, $21.2 \%$ and $30.8 \%$ of the study and control groups respectively were smoker and 32.7 and $26.9 \%$ of study and control groups respectively were exsmoker.

Figure (2) Percentage distribution regarding the total pre-post and follow up satisfactory level of knowledge among study and control group subjects showed that there were no a statistical significant difference between control and study group subjects pre- guidelines implementation regarding patient's total knowledge. While highly statistically significant difference was found between both groups post and follow up program implementation in total knowledge $(\mathrm{p}<0.001)$.

Table (3) Percentage distribution of satisfactory level of knowledge among study and control group subjects pre-post and follow up guidelines implementation displayed that, half of study and control group (50.0\% and 52.7\%, respectively) had knowledge about symptoms of CAD preprogram implementation while majority of study group $(90.4 \%, 88.4 \%),(82.7 \%)$, (90.4\%, 84.4\%), (84.4\%), (90.4\%) and $(84.4 \%, 75.8 \%)$ respectively had knowledge about risk factors, symptoms, diet, exercise, smoking and sexual relation of CAD post and follow up guidelines intervention implementation with a highly statistically significant difference was found between both groups post and follow up guidelines intervention implementation regarding all items of knowledge except knowledge about symptoms.

There was improvement through phases of the study (pre to post and follow up implementation phases) regarding all items of knowledge among study group. While concerning the control group there was no improvement.

Table (4) Comparison of mean scores of somatic health complain questionnaire (breathlessness, fatigue, pain and unrest) 
between study and control group subjects' pre- post and follow up guidelines implementation. Regarding all dimensions of health complaints scale (Breathlessness, fatigue, pain and unrest), there were highly statistically significant differences between study group subjects as compared to control group at post and follow up guidelines implementation at $\mathrm{p}<0.001$, while there was no a statistically significant differences between both groups at pre guidelines implementation at $\mathrm{p}<0.05$.

Table (5) Comparison between the study and control group subjects pre- post and follow up program implementation regarding Beck anxiety scores. There was no statistical significant difference between study and control group subjects pre guidelines implementation $(\mathrm{p}=0.356)$. While, there was a highly statistically significant differences between the study and control group subjects post and follow up guidelines implementation regarding beck anxiety level scores $(<0.001)$ with very low anxiety level in post and follow up phases of implementing the educational guidelines for study group $(90.4 \%$ and $80.8 \%$, respectively).

Table (6) Relation between demographic characteristics and difference knowledge score between study and control group demonstrated that there was no statistically significance relation between demographic characteristics of study sample (study and control group) and difference knowledge score except with educational level in control group. 
Table (1): Demographic characteristics among the study and control group subjects

\begin{tabular}{|c|c|c|c|c|c|c|}
\hline \multirow[t]{3}{*}{ Items } & \multicolumn{4}{|c|}{ Total No $(\mathrm{Tn}=104)$} & \multirow{3}{*}{$\begin{array}{l}\text { Chi } \\
\text { square }\end{array}$} & \multirow{3}{*}{$\begin{array}{l}p \\
\text { value }\end{array}$} \\
\hline & \multicolumn{2}{|l|}{ Study $(n=52)$} & \multicolumn{2}{|l|}{ Control $(n=52)$} & & \\
\hline & No & $\%$ & No & $\%$ & & \\
\hline \multicolumn{7}{|l|}{ Age (in years) } \\
\hline \multirow{5}{*}{$\begin{array}{l}-20 \\
-30 \\
-40 \\
-50 \\
+60\end{array}$} & 0 & 0 & 1 & 1.9 & 0.274 & 0.78 \\
\hline & 3 & 5.8 & 4 & 7.7 & & \\
\hline & 9 & 17.3 & 10 & 19.23 & & \\
\hline & 28 & 53.8 & 19 & 36.5 & & \\
\hline & 12 & 23.07 & 18 & 34.6 & & \\
\hline$($ Mean \pm SD) & $54.21 \pm 8.01$ & & $54.71 \pm 10.42$ & & & \\
\hline \multicolumn{7}{|l|}{ Sex } \\
\hline \multirow{2}{*}{$\begin{array}{l}\text { Female } \\
\text { Male }\end{array}$} & 21 & 40.4 & 21 & 40.4 & 0.000 & 1.000 \\
\hline & 31 & 59.6 & 31 & 59.6 & & \\
\hline \multicolumn{7}{|l|}{ Residence } \\
\hline \multirow{2}{*}{$\begin{array}{l}\text { Rural } \\
\text { Urban }\end{array}$} & 8 & 15.4 & 17 & 32.7 & 4.265 & 0.039 \\
\hline & 44 & 84.6 & 35 & 67.3 & & \\
\hline \multirow{5}{*}{$\begin{array}{l}\text { Educational level } \\
\text { Illiterate } \\
\text { Read \& Write } \\
\text { Intermediate education } \\
\text { High education }\end{array}$} & & & & & & \\
\hline & 15 & 28.8 & 15 & 28.8 & 1.209 & 0.751 \\
\hline & 23 & 44.2 & 27 & 51.9 & & \\
\hline & 11 & 21.2 & 7 & 13.5 & & \\
\hline & 3 & 5.8 & 3 & 5.8 & & \\
\hline \multirow{5}{*}{$\begin{array}{l}\text { Occupation } \\
\text { Employee } \\
\text { Muscular work } \\
\text { Retired } \\
\text { No work }\end{array}$} & & & & & & \\
\hline & 5 & 9.6 & 3 & 5.8 & 1.209 & 0.751 \\
\hline & 14 & 26.9 & 18 & 34.6 & & \\
\hline & 10 & 19.2 & 7 & 13.5 & & \\
\hline & 23 & 44.2 & 24 & 46.2 & & \\
\hline
\end{tabular}


Not significant (NS) $\mathrm{p}>\mathbf{0 . 0 5}$

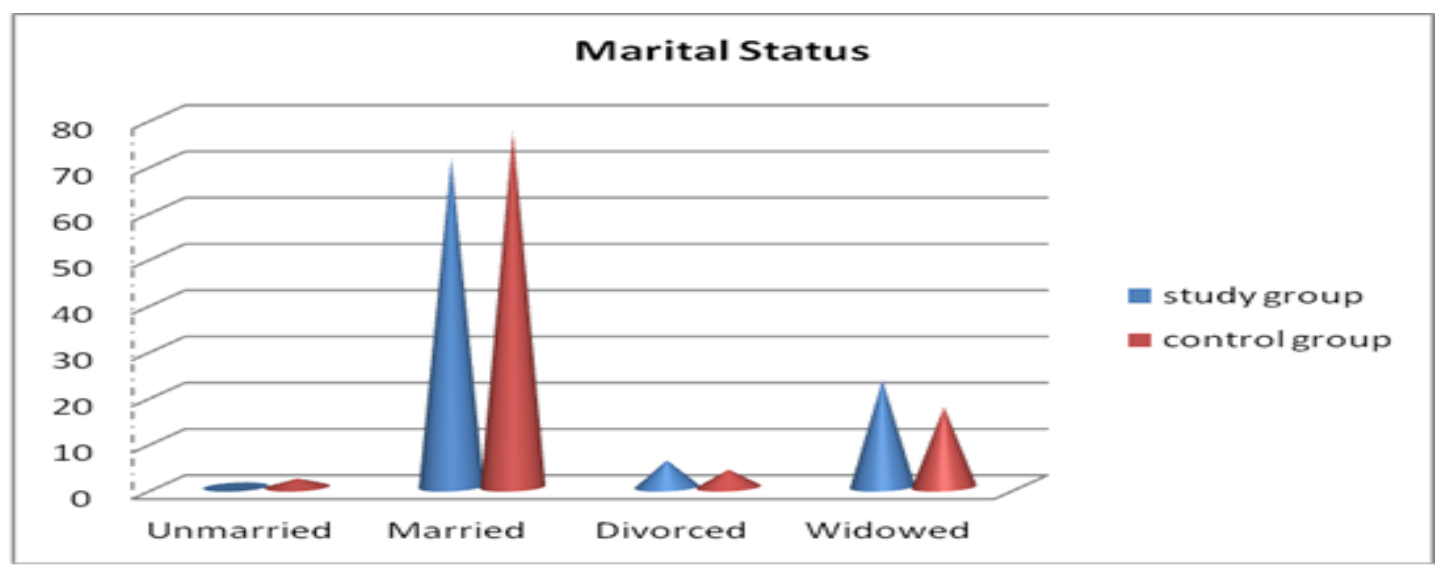

Figure (1): Percentage distribution of the studied groups by marital status

Table (2): Percentage distribution among the study and control group subjects as regards health history

\begin{tabular}{|c|c|c|c|c|c|c|}
\hline \multirow[t]{3}{*}{ Items } & \multicolumn{4}{|c|}{ Total No $(\mathrm{N}=104)$} & \multirow[t]{3}{*}{ Chi square } & \multirow[t]{3}{*}{ p value } \\
\hline & \multicolumn{2}{|c|}{ Study $(n=52)$} & \multicolumn{2}{|c|}{ Control(n=52) } & & \\
\hline & No & $\%$ & No & $\%$ & & \\
\hline Diabetes & 22 & 42.3 & 25 & 48.1 & 0.349 & 0.554 \\
\hline Hypertension & 38 & 73.1 & 40 & 76.9 & 0.205 & 0.651 \\
\hline Angina & 31 & 59.6 & 33 & 63.5 & 0.163 & 0.687 \\
\hline IHD & 14 & 26.9 & 27 & 51.9 & 6.804 & 0.009 \\
\hline Liver cirrhosis & 10 & 19.0 & 14 & 26.9 & 0.369 & 0.54 \\
\hline COPD & 8 & 15.4 & 7 & 13.5 & & \\
\hline Cardiac catheterization & 13 & 25.0 & 19 & 36.5 & 1.625 & 0.202 \\
\hline Open heart surgery & 1 & 1.9 & 2 & 3.8 & 0.343 & 0.558 \\
\hline \multicolumn{7}{|c|}{ Compliance with medication } \\
\hline No & 1 & 1.9 & 5 & 9.6 & \multirow{3}{*}{2.965} & \multirow{3}{*}{0.227} \\
\hline Incomplete Compliance & 42 & 80.8 & 40 & 76.9 & & \\
\hline Complete Compliance & 9 & 17.3 & 7 & 13.5 & & \\
\hline \multicolumn{7}{|l|}{ Smoking } \\
\hline No & 24 & 46.2 & 22 & 42.3 & \multirow{3}{*}{\multicolumn{2}{|c|}{$\begin{array}{l}1.303 \\
0.521\end{array}$}} \\
\hline Yes & 11 & 21.2 & 16 & 30.8 & & \\
\hline Ex-smoker & 17 & 32.7 & 14 & 26.9 & & \\
\hline
\end{tabular}




\section{The total pre-post and follow up test of satisfactory scores of knowledge}

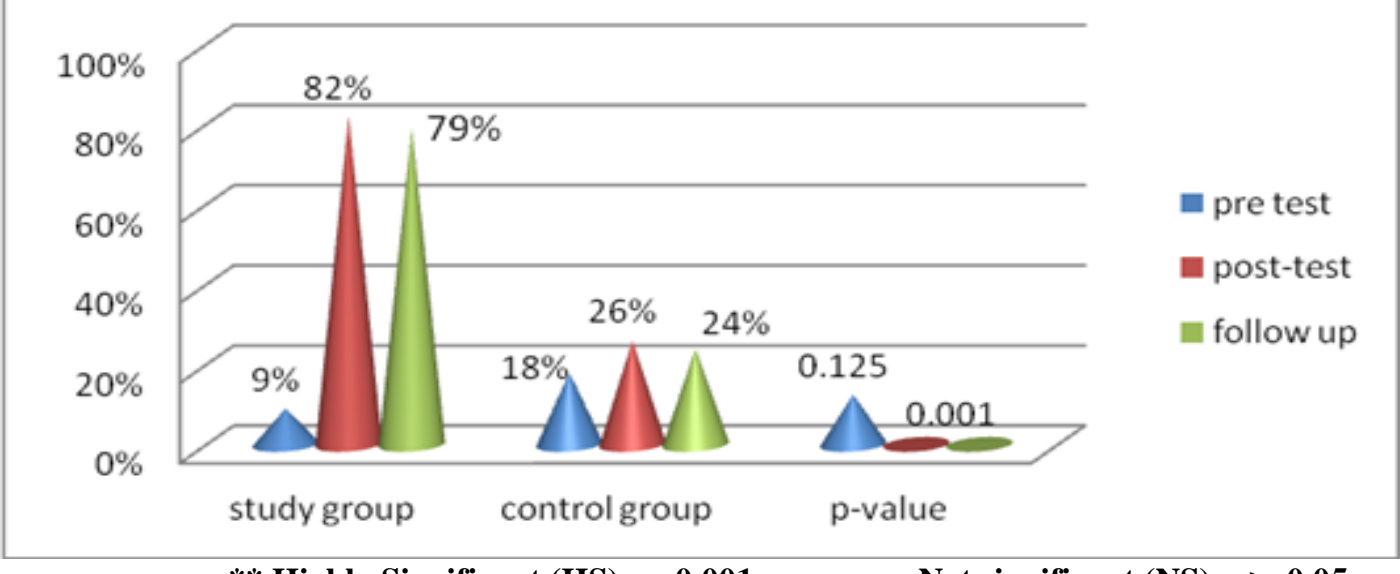

** Highly Significant (HS) p $<0.001 \quad$ Not significant (NS) p $>0.05$

Fig (2): Percentage distribution regarding the total pre-post and follow up satisfactory level of knowledge among study \& control group subjects. 
Table (3): Percentage distribution of satisfactory level of knowledge among study and control group subjects pre-post and follow up guidelines implementation

\begin{tabular}{|c|c|c|c|c|c|c|c|c|c|c|c|c|}
\hline \multirow{3}{*}{ Items } & \multicolumn{4}{|c|}{ Pre- test } & \multicolumn{4}{|c|}{ Post- test } & \multicolumn{4}{|c|}{ owFoll- test up after three months } \\
\hline & $\begin{array}{l}\text { Study } \\
\text { group }\end{array}$ & $\begin{array}{c}\text { Control } \\
\text { group }\end{array}$ & Chi & p & $\begin{array}{l}\text { Study } \\
\text { group }\end{array}$ & $\begin{array}{c}\text { Control } \\
\text { group }\end{array}$ & Chi & p value & $\begin{array}{l}\text { Study } \\
\text { group }\end{array}$ & $\begin{array}{c}\text { Control } \\
\text { group }\end{array}$ & Chi & p value \\
\hline & $\%$ & $\%$ & & & $\%$ & $\%$ & & & $\%$ & $\%$ & & \\
\hline Definition & 5.8 & 21.2 & 5.283 & 0.022 & 75.0 & 21.2 & 30.199 & $\begin{array}{c}<0.001 * \\
*\end{array}$ & 73.0 & 20.2 & 30.99 & $\begin{array}{c}<0.001 * \\
*\end{array}$ \\
\hline Risk factors & 0.0 & 3.8 & 2.039 & 0.153 & 90.4 & 3.8 & 78.145 & $\begin{array}{c}<0.001 * \\
*\end{array}$ & 88.4 & 3.8 & 79.845 & $\begin{array}{c}<0.001 * \\
*\end{array}$ \\
\hline Symptoms & 50.0 & 52.7 & 12.446 & $\begin{array}{c}<0.00 \\
1 * *\end{array}$ & 82.7 & 64.6 & 0.07 & 0.791 & 82.7 & 60.6 & 0.09 & 0.661 \\
\hline Diagnosis & 3.8 & 3.8 & 0 & 1 & 55.8 & 3.8 & 33.502 & $\begin{array}{c}<0.001 * \\
*\end{array}$ & 55.8 & 3.8 & 33.502 & $\begin{array}{c}<0.001 * \\
*\end{array}$ \\
\hline Treatment & 0.0 & 0.0 & 0 & 1 & 75.0 & 11.5 & 35.199 & $\begin{array}{c}<0.001 * \\
*\end{array}$ & 36.5 & 0.0 & 23.247 & $\begin{array}{c}<0.001 * \\
*\end{array}$ \\
\hline Diet & 3.8 & 0.0 & 2.039 & 0.153 & 90.4 & 3.8 & 78.145 & $\begin{array}{c}<0.001 * \\
*\end{array}$ & 84.4 & 3.8 & 68.215 & $\begin{array}{c}<0.001 * \\
*\end{array}$ \\
\hline Exercise & 0.0 & 0.0 & 0 & 1 & 84.4 & 3.8 & 68.215 & $\begin{array}{c}<0.001 * \\
*\end{array}$ & 84.4 & 0.0 & 58.335 & $\begin{array}{c}<0.001 * \\
*\end{array}$ \\
\hline Smoking & 3.8 & 3.8 & 0 & 1 & 90.4 & 3.8 & 78.145 & $\begin{array}{c}<0.001 * \\
*\end{array}$ & 90.4 & 3.8 & 78.145 & $\begin{array}{c}<0.001 * \\
*\end{array}$ \\
\hline $\begin{array}{l}\text { Sexual } \\
\text { relation }\end{array}$ & 0.0 & 0.0 & 0 & 1 & 84.4 & 3.8 & 68.215 & $\begin{array}{c}<0.001 * \\
*\end{array}$ & 75.8 & 0.0 & 32.022 & $\begin{array}{c}<0.001 * \\
*\end{array}$ \\
\hline
\end{tabular}

** Highly Significant (HS) $<0.001$

Not significant (NS) $p>0.05$

Table (4): Comparison of Mean scores of Somatic Health Complain Questionnaire (Breathlessness,

Fatigue, Pain and unrest) between study and control group subjects pre- post \& follow up guidelines implementation

\begin{tabular}{|c|c|c|c|c|c|c|c|c|c|c|c|c|}
\hline \multirow{3}{*}{$\begin{array}{l}\text { Items } \\
\text { SHCQ }\end{array}$} & \multicolumn{4}{|c|}{ Pre- test } & \multicolumn{4}{|c|}{ Post- test } & \multicolumn{4}{|c|}{ Follow- up test after three months } \\
\hline & $\begin{array}{l}\text { Study } \\
\text { group }\end{array}$ & $\begin{array}{l}\text { Contro } \\
1 \text { group }\end{array}$ & & & $\begin{array}{l}\text { Study } \\
\text { group }\end{array}$ & $\begin{array}{c}\text { Control } \\
\text { group }\end{array}$ & & & $\begin{array}{l}\text { Study } \\
\text { group }\end{array}$ & $\begin{array}{c}\text { Control } \\
\text { group }\end{array}$ & & $\mathbf{p}$ \\
\hline & $\begin{array}{c}\text { Mean } \\
\pm \text { SD }\end{array}$ & $\begin{array}{c}\text { Mean } \\
\pm \text { SD }\end{array}$ & $\begin{array}{c}\text { t- } \\
\text { test }\end{array}$ & $\begin{array}{c}\text { p } \\
\text { value }\end{array}$ & $\begin{array}{r}\text { Mean } \\
\pm \text { SD }\end{array}$ & $\begin{array}{r}\text { Mean } \\
\pm \text { SD }\end{array}$ & t-test & $\begin{array}{c}\text { p } \\
\text { value }\end{array}$ & $\begin{array}{r}\text { Mean } \\
\pm \text { SD }\end{array}$ & $\begin{array}{r}\text { Mean } \\
\pm \text { SD }\end{array}$ & t-test & $\begin{array}{c}\text { valu } \\
\text { e }\end{array}$ \\
\hline Breathlessness & $\begin{array}{c}8.87 \pm \\
1.79\end{array}$ & $\begin{array}{c}9.12 \pm \\
2.03\end{array}$ & .666 & .507 & $\begin{array}{c}4.85 \pm \\
1.43\end{array}$ & $\begin{array}{c}7.87 \pm \\
1.72\end{array}$ & 9.740 & $\begin{array}{c}<0.001 \\
* *\end{array}$ & $\begin{array}{c}4.94 \pm \\
1.30\end{array}$ & $\begin{array}{c}7.92 \pm \\
1.71\end{array}$ & 9.125 & $\begin{array}{l}<0.0 \\
01 * *\end{array}$ \\
\hline Fatigue & $\begin{array}{c}15.73 \pm \\
2.13\end{array}$ & $\begin{array}{c}16.87 \pm \\
2.59\end{array}$ & $\begin{array}{c}2.43 \\
8\end{array}$ & .016 & $\begin{array}{c}8.52 \pm \\
1.97\end{array}$ & $\begin{array}{c}14.98 \pm \\
2.50\end{array}$ & $\begin{array}{c}14.64 \\
9\end{array}$ & $\begin{array}{c}<0.001 \\
* *\end{array}$ & $\begin{array}{c}8.79 \pm \\
1.93\end{array}$ & $\begin{array}{c}15.46 \pm \\
2.19\end{array}$ & 15.019 & $\begin{array}{l}<0.0 \\
01 * *\end{array}$ \\
\hline Pain & $\begin{array}{l}17.90 \\
\pm 3.21\end{array}$ & $\begin{array}{c}17.65 \pm \\
2.43\end{array}$ & $\begin{array}{c}- \\
.448\end{array}$ & .655 & $\begin{array}{c}7.17 \pm \\
2.86\end{array}$ & $\begin{array}{c}15.25 \pm \\
2.06\end{array}$ & $\begin{array}{c}16.53 \\
0\end{array}$ & $\begin{array}{c}<0.001 \\
* *\end{array}$ & $\begin{array}{c}7.44 \pm \\
2.86\end{array}$ & $\begin{array}{c}15.38 \pm \\
2.07\end{array}$ & 16.120 & $\begin{array}{l}<0.0 \\
01 * *\end{array}$ \\
\hline Unrest & $\begin{array}{c}12.56 \pm \\
1.61\end{array}$ & $\begin{array}{c}13.87 \pm \\
1.44\end{array}$ & $\begin{array}{c}4.35 \\
7\end{array}$ & $\begin{array}{c}<0.001 \\
* *\end{array}$ & $\begin{array}{c}6.77 \pm \\
1.79\end{array}$ & $\begin{array}{c}12.13 \pm \\
1.68\end{array}$ & $\begin{array}{c}15.76 \\
2\end{array}$ & $\begin{array}{c}< \\
0.001 * \\
*\end{array}$ & $\begin{array}{c}6.77 \pm \\
1.74\end{array}$ & $\begin{array}{c}12.15 \pm \\
1.67\end{array}$ & 15.662 & $\begin{array}{c}< \\
0.00 \\
1 * *\end{array}$ \\
\hline
\end{tabular}

** Highly Significant (HS) p $<0.001$

Not significant (NS) $p>0.05$ 
Table (5): Comparison between the study and control group subjects pre- post $\&$ follow up program implementation regarding Beck anxiety scores.

\begin{tabular}{|c|c|c|c|c|c|c|}
\hline \multirow[b]{3}{*}{ Items } & \multicolumn{4}{|c|}{ Group } & \multirow{3}{*}{$\begin{array}{l}\text { Chi } \\
\text { square }\end{array}$} & \multirow[b]{3}{*}{ p value } \\
\hline & \multicolumn{2}{|c|}{ Study } & \multicolumn{2}{|c|}{ Control } & & \\
\hline & No & $\%$ & No & $\%$ & & \\
\hline \multirow{3}{*}{$\begin{array}{l}\text { Beck anxiety (pre) } \\
\text { Very low anxiety } \\
\text { Moderate anxiety } \\
\text { Persistent and high anxiety }\end{array}$} & 0 & $0.0 \%$ & 2 & $3.8 \%$ & \multirow[b]{3}{*}{2.067} & \multirow[b]{3}{*}{0.356} \\
\hline & 21 & $40.4 \%$ & 21 & $40.4 \%$ & & \\
\hline & 31 & $59.6 \%$ & 29 & $55.8 \%$ & & \\
\hline \multirow{3}{*}{$\begin{array}{l}\text { Beck anxiety (post) } \\
\text { Very low anxiety } \\
\text { Moderate anxiety } \\
\text { Persistent and high anxiety }\end{array}$} & 47 & $90.4 \%$ & 7 & $13.5 \%$ & \multirow[b]{3}{*}{62.571} & \multirow[b]{3}{*}{$<0.001$} \\
\hline & 5 & $9.6 \%$ & 29 & $55.8 \%$ & & \\
\hline & 0 & $0.0 \%$ & 16 & $30.8 \%$ & & \\
\hline \multirow{3}{*}{$\begin{array}{l}\text { Beck anxiety ( follow up after three } \\
\text { months) } \\
\text { Very low anxiety } \\
\text { Moderate anxiety } \\
\text { Persistent and high anxiety } \\
\end{array}$} & 42 & $80.8 \%$ & 5 & $9.6 \%$ & \multirow[b]{3}{*}{55.254} & \multirow[b]{3}{*}{$<0.001$} \\
\hline & 8 & $15.4 \%$ & 22 & $42.3 \%$ & & \\
\hline & 2 & $3.8 \%$ & 25 & $48.1 \%$ & & \\
\hline
\end{tabular}

** Highly Significant (HS) p $<$ 0.001 Not significant (NS) p $>0.05$

Table (6): Relation between demographic characteristics and difference knowledge score between study and control group

\begin{tabular}{|c|c|c|c|c|c|c|c|c|}
\hline \multirow{4}{*}{ Items } & \multicolumn{8}{|c|}{ Difference knowledge score } \\
\hline & \multicolumn{8}{|c|}{ Group } \\
\hline & \multicolumn{4}{|c|}{ Study } & \multicolumn{4}{|c|}{ Control } \\
\hline & Mean & SD & F ratio & Significance & Mean & SD & F ratio & Significance \\
\hline $\begin{array}{c}\text { Age group } \\
<50 \\
50+\end{array}$ & $\begin{array}{l}54.2 \\
51.1\end{array}$ & $\begin{array}{c}9.9 \\
13.1\end{array}$ & 1.152 & 0.234 & $\begin{array}{c}1.1 \\
.2\end{array}$ & $\begin{array}{l}2.2 \\
2.2\end{array}$ & 0.966 & 0.468 \\
\hline $\begin{array}{l}\text { Educational level } \\
\text { Illiterate } \\
\text { Read and write } \\
\text { High school } \\
\text { University }\end{array}$ & $\begin{array}{l}49.9 \\
52.8 \\
62.7 \\
56.5\end{array}$ & \begin{tabular}{|c|}
8.4 \\
10.2 \\
13.2 \\
11.5
\end{tabular} & 0.941 & 0.606 & $\begin{array}{l}.6 \\
.9 \\
.8 \\
.0\end{array}$ & $\begin{array}{l}1.5 \\
1.8 \\
3.3 \\
4.3\end{array}$ & 3.145 & 0.009 \\
\hline
\end{tabular}




\section{Discussion}

Coronary artery disease is one of the most common cardiovascular diseases in adults.

The disease is one of the most preventable non-communicable diseases and the use of primary and secondary prevention methods are key strategies in prevention of coronary artery disease. Since, teaching today to the patient is recognized as one of the main duties of nurses, training in heart disease and related care is essential. In this regard, the optimal selection educational methods that lead to increased awareness are significant ${ }^{(27)}$.

\section{Regarding demographic characteristics} of patients under study, the results of the present study revealed that more than half of study group and about one third of control group subjects were in the same age group from 50 years to less than 60 years old with mean age (54.21 \pm 8.01$)$ and (54.71 \pm 10.42 ) respectively). This result is in accordance with Moeini, et al., (2020) ${ }^{(27)}$, who studied the effect of aromatherapy on the quality of sleep in ischemic heart disease patients and found that the mean and SD of patients' age was $55.7 \pm 7.7$ in the study group and $52.8 \pm 8.5$ for the control .In the same context Roman et al. $\mathbf{( 2 0 1 9 )}^{(28)}$, found that more than half of the patients were within the age group of 45 years and above, while only $19 \%$ were less 45 years. As well Nematollahi, et al., $(\mathbf{2 0 1 7})^{(29)}$, found that the mean age in their study was $50 \cdot 33 \pm 12 \cdot 14$ years. This mean age of the present study may be due to aging which is an un-modifiable risk factor for $\mathrm{CAD}$ and the WHO reports that CAD risk increase as age increases.

Concerning sex the present study clarified that more than half of study and control group subjects were males. This result is supported by Moeini et al. (2020) ${ }^{(28)}$, who stated that $65.6 \%$ and $59.4 \%$ in the study and control group respectively were male. However this result is inconsistent with Farshid el al., (2020) ${ }^{(30)}$, in a study titled the role of gender in the importance of risk factors for coronary artery disease, that two thirds of study subjects were female and one third were male.

Concerning the residence, the current study results showed that the majority of the study group and slightly more than two thirds of control group were living in urban areas. This is supported by Mahmood et al., (2017) $^{(31)}$ in the study of effect of aromatherapy on anxiety in patients with acute coronary syndrome hospitalized in cardiac care unit and concluded that two thirds of study and control group reside in urban area. This result is incongruent with Abdul-Hasan and Kathim (2018) ${ }^{(32)}$ who mentioned that the majority of the studied subjects lived in rural area and only onefourth lived in urban area. 
As regard marital status in the current study, the results showed that about two-thirds of the study and control group were married. This finding goes in line with Abdullah and Baker (2019) ${ }^{(33)}$, in a paper entitled health beliefs of patients with coronary heart disease toward secondary prevention and mentioned that about nearly all sample $(98.4 \%)$ were married.

Concerning educational level, the study results revealed that less than half of the study group and about half of control group subjects can read and write. This result was in an agreement with the findings of the study had done by Al-Abbudi et al. (2018) $^{(34)}$, to determine the incidence and to estimate the socio-demographical characteristics of CHD patients in Baghdad Teaching Hospital, who reported that about more than two thirds of the patients had low educational level and can't read and write.

While this result is inconsistent with Subramanian (Ahmed et al., 2019) ${ }^{(35)}$, in a study of the pattern of risk-factor profile in Egyptian patients with acute coronary syndrome and mentioned that nearly half of their studied subjects had high education, with $17 \%$ reporting no education.

Regarding patient's occupation, the present study result indicated that, less than half of study and control group subjects were not working. This may be due increasing age level among the studied subjects and the fact that $\mathrm{CAD}$ patients refrained of job due to activity intolerance. This result in agreement with Abbasi, et al., (2018) ${ }^{(36)}$, in a study of designing and manufacturing of educational multimedia software for preventing coronary artery disease whom mentioned that less than fifth of their study were employee .While this result is inconsistent with Eid (2018) ${ }^{(37)}$, who mentioned that more than half of the sample was working.

The results showed also, that there were no statistically significant differences between study and control group subjects regarding all aspects of socio-demographic characteristics at $\mathrm{p}<0.05$. This indicated that both study and control groups are compatible.

Considering health history, the present study indicated that less than half of study group and control group subjects had diabetes mellitus. This may be due to that the majority of the patients were within 3050 years and this age group commonly is high risk for D.M. This result is in accordance with Ramadhani et al., (2019) (36) in a study of investigating the relevance of nursing caring interventions delivered to patients with coronary artery disease and found that diabetes mellitus as comorbidity for CAD existed in almost three-quarter of patients . In the same context Madonna et al. (2019) $)^{(39)}$, in a study entitled impact of 
sex differences and diabetes on coronary atherosclerosis and ischemic heart disease reported that the cardiovascular hazard in people with diabetes is higher from two to three times than those without the disease. On the other hand, this finding is disagreement with Salari et al. (2018) $)^{(40)}$, who stated that the majority of their study subjects had no diabetes.

In the same line, the present study indicated that more than two thirds of study and control group subjects had hypertension. This may be due to that hypertension is considered predisposing factor for CAD. This result is in accordance with Hassan et al. (2020) $)^{(41)}$ in a study of dietary rehabilitation effectiveness on coronary artery diseases patient's outcomes who mentioned that majority of the subjects had a history of hypertension. Also this is congruent with Roman et al. (2019) (29) who studied the assessment of risk factors for cardiovascular diseases among patients attending cardiac clinic and found that two thirds of the study subjects were clinically diagnosed with hypertension. As well Farshid et al. (2020) ${ }^{(\mathbf{4 0})}$, in a study titled the role of gender in the importance of risk factors for coronary artery disease found that hypertension was the most influential risk factor in the study. In the same line Abdullah and Baker (2019) ${ }^{(32)}$, in a study of health beliefs of patients with coronary heart disease toward secondary prevention reported that slightly more than half of the studied subjects had suffered from diabetes and hypertension respectively.

Concerning history of angina the present study finding showed that more than half of study and more than two thirds of control groups had angina. This result goes in the same line with Gecaite-Stonciene et al. $(\mathbf{2 0 2 0})^{(42)}$, who reported that about half of the sample was diagnosed with stable angina, As well the findings were supported by Hassan, et al. (2020) ${ }^{(41)}$, who stated that two thirds and slightly more than half of study and control group had angina.

As regards history of cardiac catheterization the present study findings showed that one fourth of the study group and more than one third of control group subjects had previous cardiac catheterization. This may be due to that most CAD patients experience cardiac catheterization as a routine investigation for diagnosis of CAD. In this context Ghisi et al. (2020) $)^{(43)}$, in a study of effectiveness of an education intervention among cardiac rehabilitation patients in Canada and stated that near than half of their study subjects performed cardiac catheterization.

Concerning compliance with medication the majority of study and control group respectively had incomplete compliance with medication, this findings goes in the same line with a study carried out by 
Khatib et al. (2019) ${ }^{(44)}$, on a study of adherence to coronary artery disease secondary prevention medicines: exploring modifiable barriers and found that half of study subjects was non-adherence patients with CAD medications.

In the same context Salari et al. (2018) $)^{(40)}$, in a study of medication adherence and its related factors in patients undergoing coronary artery angioplasty reported that about a third of the participants were nonadherence to medications. This could be due forgetfulness of study subjects, worry that their medicines will do more harm than good and lack of sufficient training. Those were the most common reasons for nonadherence as stated by patients.

Concerning smoking, one fifth and one third of study and control groups respectively were smoker and one third and one fourth of study and control groups respectively were ex-smoker. This result goes in the same line with Hassan, et al. $\mathbf{( 2 0 2 0}^{(41)}$, who reported that less than half and around one third of study and control group were smoker. While this result is in disagreement with Egyptian study carried out by Ahmed et al. (2019) ${ }^{(35)}$, who mentioned that of majority of men presenting in the study, were smokers and as and ex-smokers $(74 \%$ were current smokers $\& 12 \%$ were ex-smokers. This may indicate that smoking is considered a major risk factor for coronary artery disease.

Regarding knowledge among study and control group subjects at pre, post and follow up guidelines implementation ,the results of the current study revealed that there were no statistical significant differences between study and control group subjects pre- guidelines implementation regarding patient's total mean scores of knowledge. Also, the results of the current study supported the current study hypothesis as a highly statistically significant differences were found between study and control groups regarding patient's total mean scores of knowledge at post and follow up guidelines implementation phases.

The improvement of patient's knowledge may be as a result of guidelines implementation as well as the permissive atmosphere of conduction, also guidelines intervention were appropriate to the individual in terms of gender, age, culture, and socioeconomic factors, as these factors have an important impact on the ability of individuals to learn. In addition, the using of open discussion during the application of the educational guidelines and giving them actual chance to participate as well giving written information may have contributed to the success of the intervention. Such 
approaches have been recognized as being important when performing patient education sessions; this explanation is supported by Ghisi et al., (2020) ${ }^{(43)}$.

This result goes on line with Tawalbeh and Ahmad (2015) ${ }^{(45)}$ in the study of the effect of cardiac education on knowledge and adherence to healthy lifestyle and proved that the coronary heart disease educational program significantly increased knowledge of the patients with heart disease. As well the study result is congruent with Mohamad et al., (2018) ${ }^{(46)}$, in a study entitled the effectiveness of health education program on knowledge of coronary heart disease who that revealed there was a statistically significance different between pre and post- knowledge level in the study.

In the same context Ghisi et al. (2020) $)^{(43)}$, demonstrated similar results in a study of the effectiveness of an Education Intervention among Cardiac Rehabilitation Patients and found that there was a significant improvement in patients' overall knowledge pre- to post cardiac rehabilitation.

When the effect of guidelines implementation on the knowledge items was examined, a highly statistically significant difference was found between both groups post and follow up program implementation regarding almost all items of knowledge. The result indicated a significant improvement of general knowledge, treatment, diet, exercise, smoking and sexual relation of CAD post and follow up program. These findings are in accordance with Clark et al. (2015) ${ }^{(47)}$, which showed that signs and symptoms and treatment were ranked as the most important items learn among patients with cardiac disease. This finding, could be attributed to patients' perceptions of the importance of improving knowledge about treatment and symptoms.

Regarding the effect of the educational guidelines on health complaints, as regard all dimensions of health complaints scale (Breathlessness, fatigue, pain and unrest), there were a highly statistically significant differences between study group subjects as compared to control group at post and follow up guidelines implementation at $\mathrm{p}<$ 0.001 , while there was no statistically significant differences between both groups at pre guidelines implementation at $\mathrm{p}<$ 0.05, which support the current study hypothesis. This may be due to effect of the educational guidelines implementation in reducing the four dimensions of health complaints in CAD patients. This result is consistent with the results of Skodova et al. (2010) $^{(48)}$, who found that there were highly statistically significant differences between study and control group subjects 
after program implementation phase regarding four dimensions of SHCQ.

On the same line the study conducted by Rogers et al., (2021) ${ }^{(49)}$, about identifying and managing functional cardiac symptoms found that common symptoms include noncardiac chest pain, fatigue shortness of breath and palpitations and there is substantial variation in presentation and severity of symptoms ranging from persistent symptoms, severe distress to minor distress or concern with resolution of symptoms before and after program implementation. In the same context Wang et al. $(\mathbf{2 0 1 5})^{(50)}$, mentioned that the intervention group exhibited a significantly greater decrease in the level of fatigue compared with the control group, whom exhibited no significant changes. Bunevicius, et al., (2013) ${ }^{(51)}$, in a research article titled "Relationship of fatigue and exercise capacity with emotional and physical state in patients with coronary artery disease" that there were highly statistically significant differences between study and control group subjects post program implementation.

Regarding anxiety level, it's noticed from the finding of the current study that most of study group had very low anxiety level in post and follow up phases of implementing the educational program. This could be due to effectiveness of educational program in reducing anxiety. The results also revealed that there were highly statistically significant differences between study and control group subjects as regard anxiety level scores post program implementation. In the same line a study conducted by Moghimian, et al., (2019) ${ }^{(52)}$, stated that there was a significant difference in the mean score between the intervention and the control group after program intervention. This could be explained as enriching patients with knowledge about their disease and managing symptoms as well improving physical condition and modifying health behaviors by different coping strategies having a positive effect in reducing anxiety level of patients.

The study findings are in contrast with Bendig (2021) ${ }^{(53)}$, in study about lessons learned from an attempted randomizedcontrolled feasibility trial for people living with coronary artery disease who revealed that there was no significant change over time in anxiety level $(\mathrm{p}>0.05)$.

As regards relation between knowledge level and demographic characteristics including age and educational level, the study findings showed that there was no statistically significance relation between demographic characteristics of study sample and knowledge level except with educational level in control group. This result is congruent with Abdullah and 
Baker (2019) ${ }^{(33)}$, in a study of health beliefs of patients with coronary heart disease toward secondary prevention and found a highly significant association between the patients' knowledge with their educational level and there is a no significant association between the patients' knowledge and their age groups. On the same line Mohamad et al. (2018) ${ }^{(46)}$ concluded that there was a relationship between knowledge level and education level.

\section{Conclusion}

Based on the findings of the present study, it can be concluded that: There were a highly statistically significant improvement regarding the mean scores of total knowledge, health complaints and anxiety level for the study group at the post and follow up phases of the educational guidelines implementation. As compared to control group, there were no statistically significant differences between total prepost and follow up test scores of knowledge, health complaints and anxiety level. The results of the current study supported the hypothesis of the study that, there were statistically significant differences between study and control groups in relation to knowledge, health complaints and anxiety level at post and follow up guidelines implementation. These findings concluded that educational guidelines had a positive effect on patient knowledge, health complaints and anxiety level.

\section{Recommendations}

Based on the current study findings;

- Future researches are prerequisite to develop telecommunication interventions (telephone, internet, and videoconference) in secondary prevention for patients with CAD to promote effective reduction of risk factors and may increase survival.

- Future researches are requisite to develop and enhance interventions to improve patient's compliance to treatment and prevent further deterioration.

- Develop and coordinate a multidisciplinary team approach in cardiology unit that contains qualified nurse, cardiology, psychologist and social worker to provide optimal and comprehensive care for patients.

- Develop systematically continuous self-management programs for patients with CAD to help improving health status and maintaining a life style at highest possible level of quality of life.

\section{References}

1. Ralapanawa, U, Sivakanesan, R. Epidemiology and the Magnitude of 
Coronary Artery Disease and Acute Coronary Syndrome: A Narrative Review; Journal of Epidemiology and Global Health; 2021; 11(2); 169 - 177.

2. Brown J, Gerhardt T, Kwon E. Risk Factors for Coronary Artery Disease; Stat Pearls; June 6, 2020. Available from:

https://www.ncbi.nlm.nih.gov/books/N BK554410.

3. Bashandya M, Abd Elgalil H, Abou Elhassan H. Epidemiological and clinical profile of acute coronary syndrome of Egyptian patients admitted to the Coronary Care Unit, Al-Azhar University Hospital, New Damietta, The Scientific Journal of AlAzhar Medical Faculty, Girls; 2019; Vol. 3 No. 3.

4. Bhosale S. The Role of Lifestyle in Development of Coronary Heart Disease; Inflammatory Heart Diseases;, IntechOpen, October 23, 2019; 306. https://www.intechopen.com/books/infl ammatory-heart-diseases.

5. Shahjehan R, Bhutta BS. Coronary Artery Disease: Stat Pearls; Feb 7, 2021. Available from: https://www.ncbi.nlm.nih.gov/books/N BK564304.

6. Shah N. Coronary Artery Atherosclerosis Clinical Presentation; the heart org. Medscape; Apr 09, 2021. https://emedicine.medscape.com/article /153647-clinical.

7. Malinauskas R, Malinauskaiene V. Self-reported physical inactivity and health complaints: a cross-sectional study of Lithuanian adolescent schoolgirls; 2016. Available at http://www.scielo.br/pdf/csp/v31n5/01 02- 311X-csp-31-5-0981.

8. Burkauskas J, Lang P, Bunevičius A, Neverauskas J, Bučiūtė-Jankauskienè M, Mickuvienè N. Cognitive function in patients with coronary artery disease: A literature review; The Journal of international medical research; 2018; 46(10), 4019-4031.

9. Covello A, Horwitz L, Singhal S. Cardiovascular disease and cumulative incidence of cognitive impairment in the Health and Retirement Study; BMC Geriatr; 2021; $21,274$.

10. Murphy B, Le Grande M, Alvarenga M, Worcester M, Jackson A. Anxiety and depression after a cardiac event: prevalence and predictors. Front. Psychol.; 2020; 10:3010.

11. Wu Y, Chen Z, Duan J, Huang K, Zhu B, Yang L. Serum Levels of FGF21, $\beta$-Klotho, and BDNF in stable coronary artery disease patients with depressive symptoms: a cross-sectional 
single-center study. Front. Psychiatry; 2021; 11:587492.

12. Foldes-Busque G. Epidemiology and prognostic implications of panic disorder and generalized anxiety disorder in patients with coronary artery disease: rationale and design for a longitudinal cohort study. BMC Cardiovasc Disord; 2021; 21(26).

13. Knuuti J, et al. European Society of Cardiology (ESC) Guidelines for The Diagnosis and Management of Chronic Coronary Syndromes. European Heart Journal; 2020; 41, 407_477.

14. Bēta G. Patient Education Relevance in Nursing Education and Practice. Science and Education Publishing Journal; 2014; 2(7):436563.

15. Koren D, Sharaf F. Impact of health education on compliance among patients of chronic diseases in $\mathrm{Al}$ Qassim, Saudi Arabia, International Journal of Health Sciences; 2016;4(2): 139-148.

16. Marcus C. Strategies for improving the quality of verbal patient and family education: a review of the literature and creation of the EDUCATE model. U.S. National Library of Medicine, PubMed Journal; 2014; 2(1): 482-49.
17. Khan M. et al. Global Epidemiology of Ischemic Heart

Disease: Results from the Global Burden of Disease Study; Cureus; Jul 23, 2020; 12(7):e9349. Global Epidemiology of Ischemic Heart Disease: Results from the Global Burden of Disease Study - PubMed (nih.gov) accessed June 4, 2021.

18. World Health Organization Rankings. WHO, World Bank, UNESCO, CIA and individual country databases for global health and causes of death; Egypt Coronary Heart Disease; 2021. Available at https:// worldlifeexpectancy.com/countryhealth-profile/Egypt. Accessed; June 4, 2021.

19. World Health Organization (WHO). Global action plan for the prevention and control of noncommunicable diseases: 20132020[Internet]. Geneva: World Health Organization; [cited 2017 Apr 11]. Available from: http://apps.who.int/iris/ bit stream/10665/94384/1/9789241506236 _eng.pdf.

20. Mechanic O, Gavin M, Grossman S. Acute Myocardial Infarction (Nursing). In: Stat Pearls [Internet]. Treasure Island (FL): Stat Pearls Publishing; Mar 9, 2021. Available from: 
https://www.ncbi.nlm.nih.gov/books/N

BK568759.

21. Azadnajafabad S, Mohammadi F, Aminorroaya A. Non-communicable diseases' risk factors in Iran; a review of the present status and action plans. $\mathbf{J}$ Diabetes Metab Disord; January, 2021.

22. Song X, Ren C, Liu P, Tao L, Zhao W, Gao W. Effect of SmartphoneBased Telemonitored Exercise Rehabilitation among Patients with Coronary Heart Disease, Journal of Cardiovascular Translational Research; 2019. Available at https://doi.org/10.1007/s12265-01909938-6. Last access :( 8/7/2020 2 $\mathrm{PM})$.

23. Yu H, Zhan P, Wang X, Wang Y, Zhang B. Effect of Health Education Based on Behavioral Change Theories on Self-Efficacy and Self-Management Behaviors in Patients with Chronic Heart Failure. Iran J Public Health; 2019; 48 (3): 421-428.

24. Murfin R. Coronary heart disease knowledge and health behavior in student nurses; 2010; 17,301, PP. 123132.

25. Brink E, Cliffordson C, Herlitz J, Karlson B. Dimensions of the somatic health complaints questionnaire (SHCQ) in a sample of myocardial infarction patients. European Journal of
Cardiovascular Nursing; 2007; 6 (1); pp. 27-31.

26. Beck A, Steer R. Manual for the Beck Anxiety Inventory. San Antonio, TX: Psychological Corporation; 1990.

27. Moeini M, Khadibi M, Bekhradi R, Ahmad S, Nazari F. Effect of aromatherapy on the quality of sleep in ischemic heart disease patients hospitalized in intensive care units of heart hospitals of the Isfahan University of Medical Sciences IJNMR; 2020; 15(4): 234-239.

28. Roman W, David H, Sauli E. Assessment of risk factors for cardiovascular diseases among patients attending cardiac clinic at a referral hospital, Journal of Xiangya Medicine J Xiangya Med; 2019; vol 4.

29. Nematollahi M, Bazeli J, Moghaddam B, Aalami H. Effect of aromatherapy on anxiety in patients with acute coronary syndrome hospitalized in cardiac care unit. Bali Medical Journal; 2017; 6(2): 331-336.

30. Farshid Gh, Emami M, Hadi R, Saeid S Parastoo N. The Role of Gender in the Importance of Risk Factors for Coronary Artery Disease, Cardiology Research and Practice Journal; 2020; Volume 2020, Article ID 6527820, 6 pages. 
31. Mahmood R, Javad B, Mahdi B Hossein A. Effect of aromatherapy on anxiety in patients with acute coronary syndrome hospitalized in cardiac care unit, Bali Medical Journal; 2017; 6(2): 331-336.

32. Abdul-Hasan A, Kathim $H$. Effectiveness of an Educational Video Intervention on Anxiety Level of Patients prior to Diagnostic Coronary Catheterization in Al-Nasiriya's Cardiac Center, IOSR Journal of Nursing and Health Science (IOSRJNHS); 2018; Volume 6, Issue 4.

33. Abdullah $\mathrm{H}$, Baker $\mathrm{H}$. Health Beliefs of Patients with Coronary Heart Disease toward Secondary Prevention: The Health Beliefs Model as a Theoretical Framework, Indian Journal of Public Health Research and Development, January, 2019; Vol. 10, No. 01.

34. Al-Abbudi S, Lami F, Abed Wady

Z. Prevalence and Assessment of Severity of Depression Among Ischemic Heart Disease Patients Attending Outpatient Cardiology Department Baghdad Teaching Hospital, Baghdad, Iraq, J Psychiatry; 2018; 21(2).

35. Ahmed B, Ashraf R, Mohamed A, Soliman M, Hany R, El kersh A, Waleed A, Tamer M, Mohammed H,
Elsayed F, Khamis H. The pattern of risk-factor profile in Egyptian patients with acute coronary syndrome: Phase II of the Egyptian cross-sectional Cardio Risk project; Cardiovasc J Afr; 2019; 30.

36. Abbasi S, Mohsen S, Mehdi E, Karimian K. Designing and Manufacturing of Educational Multimedia Software for Preventing Coronary Artery Disease and Its Effects on Modifying The Risk Factors in Patients with Coronary Artery Disease, Electron J Gen Med; 2018; 15(3):em22 ISSN:2516-3507.

37. Eid M. Anxiety and depression predicted quality of life among patients with heart failure, Journal of Multidisciplinary Healthcare; 2018; 30;11:367-373.

38. Ramadhani F, Liu Y, Jing X, Qing Y, Rathnayake A, Shokat K, Wu W. Investigating the Relevance of Nursing Caring Interventions Delivered to Patients with Coronary Artery Disease at a Teaching Hospital in China: A Retrospective Study; Cureus; 2019; 11(5): e4672.

39. Madonna R, Balistreri C, De Rosa S. Impact of sex differences and diabetes on coronary atherosclerosis and ischemic heart disease, Journal of 
Clinical Medicine; 2019; vol. 8, no. 1, p. 98 .

40. Salari A, Rouhi L, Ashouri A, Moaddab F, Zaersabet F, Nourisaeed A. Medication Adherence and its Related Factors in Patients Undergoing Coronary Artery Angioplasty, journal of Caring Sciences; 2018; 7 (4): 213 218.

41. Hassan M, Eid H, Abdullah N, Shehata H, Maher, Nady S. Dietary Rehabilitation Effectiveness On Coronary Artery Diseases Patient's Outcomes, Sys Rev Pharm; 2020; 11(12):2422-2429.

42. Gecaite-Stonciene J, Bunevicius A, Burkauskas J, Brozaitiene J, Neverauskas J, Mickuviene N, Kazukauskiene N. Validation of the Multidimensional Fatigue Inventory with Coronary Artery Disease Patients, Int. J. Environ; Res. Public Health; 2020; 17; 8003.

43. Ghisi G, Rouleau F, Ross K, Monique D, Sylvie L, Crystal A, Thomas S, Paul O. Effectiveness of an Education Intervention Among Cardiac Rehabilitation Patients in Canada: A Multi-Site Study, CJC; 2020; 214- 221.

44. Khatib R, Marshall K, Silcock, J, Forrest C, Hall A. Adherence to coronary artery disease secondary prevention medicines: exploring modifiable barriers, Open Heart, BMJ; 2019; 6:e000997.

45. Tawalbeh M, Ahmad M. The Effect of Cardiac Education on Knowledge and Adherence to Healthy Lifestyle Clinical Nursing Research; 2015; 1-14.

46. Mohamad N, Ikhsan D, Ismail A, Kamaruddin A. The Effectiveness of Health Education Program on Knowledge of Coronary Heart Disease (CHD) Among Public in Puncak Alam, Selangor. Advanced Science Letters; January; 2018; Volume 24(1); pp. 556558.

47. Clark R, Fredericks B, Buitendyk N. Development and feasibility testing of an education program to improve knowledge and self-care among Aboriginal and Torres Strait Islander patients with heart failure. Rural Remote Health; 2015; 15(3):3231.

48. Skodova Z, Nagyova I, Rosenberger J, van Dijk J, Middel B, Vargova H, Sudzinova A, Studencan M, Reijneveld S. Vital exhaustion in coronary heart disease: the impact of socioeconomic status. Eur J Card Prev Rehabil; 2010; 15:572-6.

49. Rogers J, Collins G, Husain M, Docherty M. Identifying and managing functional cardiac symptoms. Clinical 
medicine (London, England); 2021;

21(1), 37-43.

50. Wang T, Huang J Chiou A. Effects

of a supportive educational nursing care programme on fatigue and quality of life in patients with heart failure: A randomized controlled trial, European Journal of Cardiovascular Nursing; $2015 ; 15(2)$.

51. Bunevicius A, Staniute M, Brozaitiene J, Neverauskas J, Bunevicius R, Relationship of fatigue and exercise capacity with emotional and physical state in patients with coronary artery disease admitted for rehabilitation program, American Heart Journal; 2013; 162(2):310-6.

52. Moghimian M, Akbari M, Moghaddasi J, Niknajad R. Effect of Digital Storytelling on Anxiety in Patients Who Are Candidates for Open-Heart Surgery Journal of Cardiovascular Nursing; 2019; Vol. 34, No. 3, pp. 231-235.

53. Bendig E, Bauereiß N, Buntrock C, Habibovic M, Ebert D, Baumeister H. Lessons learned from an attempted randomized-controlled feasibility trial on "WIDeCAD" - An internet-based depression treatment for people living with coronary artery disease (CAD); Internet Interventions; Science Direct; $2021 ; 24,100375$. 